\title{
Terapia prono y supervivencia en SARS-CoV-2 en Cuidados Intensivos de un hospital de tercer nivel de atención en México**
}

Prone therapy and survival in SARS-CoV-2 in Intensive Care of a tertiary care hospital in Mexico Terapia prona e sobrevivência em SARS-CoV-2 em terapia intensiva de um hospital de alta complexidade no México

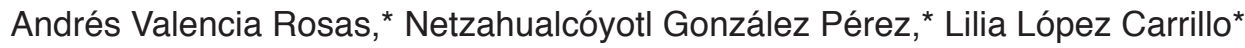

\section{RESUMEN}

Introducción: La posición en decúbito prono mejora la supervivencia en pacientes con síndrome de dificultad respiratoria aguda (SDRA) moderado o grave, por lo que es importante determinar el impacto de esta estrategia en pacientes con SARS-CoV-2.

Material y métodos: Estudio observacional, retrospectivo, longitudinal, comparativo, en pacientes con SARS-CoV-2, que requirieron ventilación mecánica por SDRA. Se calculó la probabilidad de supervivencia individual acumulada a lo largo de 60 días de seguimiento con el método de Kaplan Meier en grupos tratados con soporte multiorgánico aislado o asociado a terapia prono.

Resultados: Se reclutaron 82 pacientes, $62(75.6 \%)$ requirieron terapia prono por una relación $\mathrm{PaO}_{2} / \mathrm{FiO}_{2}<150 \mathrm{mmHg}$, mientras que 20 pacientes (24.4\%) no fueron sometidos a esta estrategia por presentar una relación $\mathrm{PaO}_{2} / \mathrm{FiO}_{2}>150 \mathrm{mmHg}$. La supervivencia a 60 días fue de 54.8 y $80 \%$, respectivamente, $(p=0.069)$.

Conclusión: La terapia prono en pacientes con SARS-CoV-2 con una relación $\mathrm{PaO}_{2} / \mathrm{FiO}_{2}<150 \mathrm{mmHg}$ es una estrategia que permite mantener una supervivencia equiparable a aquella que tienen los pacientes que ingresan con una $\mathrm{PaO}_{2} / \mathrm{FiO}_{2}$ superior a $150 \mathrm{mmHg}$.

Palabras clave: SARS-CoV-2, terapia prono, ventilación mecánica, supervivencia.

\section{ABSTRACT}

Introduction: The prone position improves survival in patients with moderate or severe acute respiratory distress syndrome (ARDS), so it is important to determine the impact of this strategy in patients with SARS-CoV-2.

Material and methods: Observational, retrospective, longitudinal, comparative study in patients with SARS-CoV-2, who required mechanical ventilation due to ARDS. The accumulated probability of individual survival was calculated over 60 days of follow-up with the Kaplan Meier method, in groups treated with isolated multiorgan support or associated with prone therapy.

Results: Eighty-two patients were recruited, 62 (75.6\%) required prone therapy due to a $\mathrm{PaO} / \mathrm{FiO}_{2}$ ratio < $150 \mathrm{mmHg}$, while 20 patients $(24.4 \%)$ were no subjected to this strategy because they presented a $\mathrm{PaO} / \mathrm{FiO}_{2}$ ratio $>150$ $\mathrm{mmHg}$. Survival at 60 days was 54.8 and $80 \%$, respectively, $(p=0.069)$.

Conclusion: Prone therapy in patients with SARS-CoV-2 with a $\mathrm{PaO}_{2} / \mathrm{FiO}_{2}$ ratio $<150 \mathrm{mmHg}$ is a strategy that allows maintaining a survival comparable to that of patients admitted with a $\mathrm{PaO}_{2} / \mathrm{FiO}_{2}$ greater than $150 \mathrm{mmHg}$.

Keywords: SARS-CoV-2, prone therapy, mechanical ventilation, survival.

\section{RESUMO}

Introdução: A posição prona melhora a sobrevida em pacientes com síndrome do desconforto respiratório agudo (SDRA) moderada ou grave, por isso é importante determinar o impacto desta estratégia em pacientes com SARS-CoV-2.

Material e métodos: Estudo observacional, retrospectivo, longitudinal e comparativo em pacientes com SARS-CoV-2, que necessitaram de ventilação mecânica por SDRA. A probabilidade acumulada de sobrevida individual foi calculada ao longo de 60 dias de acompanhamento pelo método de Kaplan-Meier, nos grupos tratados com suporte multiorgânico isolado ou associado à terapia prona.

Resultados: Oitenta e dois pacientes foram recrutados, $62(75.6 \%)$ necessitaram de terapia prona devido a uma relação $\mathrm{PaO}_{2} / \mathrm{FiO}_{2}<150 \mathrm{mmHg}$,

** Concurso Académico de Investigación «Dr. Mario Shapiro» 2020. Ganador del tercer lugar.

* Centro Médico ISSEMyM Toluca. Estado de México, México.

Recibido: 09/10/2020. Aceptado: 09/11/2020.

Citar como: Valencia RA, González PN, López CL. Terapia prono y supervivencia en SARS-CoV-2 en Cuidados Intensivos de un hospital de tercer nivel de atención en México. Med Crit. 2020;34(6):330-334. https://dx.doi.org/10.35366/98162

www.medigraphic.com/medicinacritica enquanto 20 pacientes (24.4\%) não foram submetidos a esta estratégia por apresentarem uma relação $\mathrm{PaO}_{2} / \mathrm{FiO}_{2}>150 \mathrm{mmHg}$. A sobrevivência em 60 dias foi de 54.8 e $80 \%$, respectivamente ( $p=0.069$ ).

Conclusão: A terapia prona em pacientes com SARS-CoV-2 com uma relação $\mathrm{PaO}_{2} / \mathrm{FiO}_{2}<150 \mathrm{mmHg}$ é uma estratégia que permite manter uma sobrevida comparável à de pacientes internados com $\mathrm{PaO}_{2} / \mathrm{FiO}_{2}$ maior que $150 \mathrm{mmHg}$. Palavras-chave: SARS-CoV-2, terapia prona, ventilação mecânica, sobrevivência.

\section{INTRODUCCIÓN}

El 8 de diciembre de 2019 se reportaron casos de neumonía de etiología desconocida en Wuhan, provincia de Hubei, China. Algunos pacientes desarrollaron rápidamente síndrome de dificultad respiratoria aguda (SDRA), insuficiencia respiratoria aguda y otras complicaciones graves. El 7 de enero de 2020 el Centro Chino para el Control y la Prevención de Enfermedades identificó un nuevo coronavirus que posteriormente se denominó coronavirus-19 causante de la enfermedad por coronavirus (COVID-19) capaz de generar síndrome respiratorio agudo severo (SARS-CoV-2). ${ }^{1,2}$ Tras el avance de la epidemia mundial de COVID-19 muchos pacientes desarrollaron SARS-CoV-2 y murieron debido a hipoxia grave. ${ }^{3}$

El manejo del SDRA se enfoca en el diagnóstico y tratamiento de infecciones, soporte respiratorio (incluyendo suplementación con oxígeno y ventilación con presión positiva), manejo cuidadoso de líquidos y medidas generales de apoyo como suplementos nutricionales. ${ }^{4}$ En el ensayo multicéntrico PROSEVA (Proning Severe ARDS Patients) $)^{5}$ la posición en decúbito prono mejoró la supervivencia y acortó la duración de la ventilación mecánica en comparación con la posición supina. PROSEVA reclutó sólo pacientes con SDRA moderado o grave $\left(\mathrm{PaO}_{2} /\right.$ $\mathrm{FiO}_{2}<150 \mathrm{mmHg}$ ), utilizó la posición prono al inicio de la enfermedad y durante al menos 16 horas por día en combinación con una ventilación con bajo volumen corriente: requisitos potenciales para la eficacia terapéutica. ${ }^{6}$

Al modificar la distribución regional de la presión transpulmonar, la posición en decúbito prono disminuye la heterogeneidad regional de la aireación pulmonar, lo que conduce a una mejora del intercambio de gases y a una disminución del riesgo de lesión pulmonar mecánica. ${ }^{7}$

Las sesiones largas de la posición prono ahora son recomendadas en la mayoría de los pacientes con SDRA grave. ${ }^{8}$ Sin embargo, en un estudio observa- 
cional multicéntrico, Guérin y colaboradores mostraron que sólo $13.7 \%$ de los pacientes con SDRA tuvieron esta estrategia. Incluso en pacientes con SDRA grave la tasa de uso de esta técnica fue de $32.9 \%$. Se dieron dos razones principales de la renuencia de los médicos a utilizar este método de tratamiento: 1 . Según el juicio de los médicos en la mayoría de los casos, la hipoxia en pacientes con SDRA grave no es lo suficientemente grave como para justificar el uso de la posición prono. 2. La mayoría de los pacientes con SDRA presentan inestabilidad hemodinámica, lo que impide que los médicos decidan utilizar la posición prono. ${ }^{9}$

\section{MATERIAL Y MÉTODOS}

Tipo de estudio: Observacional, ambispectivo, longitudinal, comparativo.

Diseño del estudio: Se realizó una hoja piloto de recolección de datos, la cual se llenó durante dos semanas, tiempo en que se realizaron ajustes en el orden de llenado y se incorporaron datos útiles para el propósito del estudio. Después, se procedió a recabar la información contenida en expedientes de pacientes que estuvieron hospitalizados en la $\mathrm{UCI}$ durante los siguientes cuatro meses de iniciado el estudio. Al cabo de 60 días, si el paciente ya no estaba hospitalizado, se realizó una llamada vía telefónica a su domicilio para verificar su estado.

Universo de trabajo: Expedientes clínicos de pacientes ingresados a una Unidad de Cuidados Intensivos COVID de un hospital de tercer nivel de atención en México.

Criterios de inclusión: Pacientes que ingresaron a la UCI con diagnóstico de SARS-CoV-2 con requerimiento de ventilación mecánica, con prioridad I, en el periodo comprendido de abril a julio de 2020.

Criterios de exclusión: Pacientes que no son derechohabientes e ingresaron a la $\mathrm{UCl}$ en tanto se logró el traslado a su unidad de adscripción. Pacientes que ingresaron provenientes de otras unidades de terapia intensiva. Pacientes que ingresaron a la $\mathrm{UCl}$ con una mortalidad calculada mayor a $90 \%$ de acuerdo con la escala pronóstica SOFA.

Criterios de eliminación: Pacientes cuyo expediente se encontró incompleto. Pacientes en quienes el seguimiento a 60 días no fue posible. Pacientes que permanecieron en la UCl por menos de 72 horas.

Hipótesis: La supervivencia es mayor en pacientes con SARS-CoV-2 tratados con soporte multiorgánico más terapia prono, en una Unidad de Cuidados Intensivos COVID de un hospital de tercer nivel de atención en México.

Objetivo general: Determinar qué supervivencia tienen los pacientes con SARS-CoV-2 tratados con soporte multiorgánico más terapia prono.

Objetivos específicos: 1. Determinar qué supervivencia tienen los pacientes con SARS-CoV-2 tratados con soporte multiorgánico más terapia prono en comparación con aquellos tratados únicamente con soporte multiorgánico. 2. Determinar qué supervivencia tienen los pacientes con SARS-CoV-2 en relación al tiempo en que se inicia la terapia prono como adyuvante al soporte multiorgánico. 3. Determinar qué supervivencia tienen los pacientes con SARS-CoV-2 en relación con la respuesta clínica obtenida con la terapia prono como adyuvante al soporte multiorgánico. 4. Determinar qué supervivencia tienen los pacientes con SARS-CoV-2 con soporte multiorgánico con base en el número de eventos de prono requeridos.

Operacionalización de variables: El síndrome respiratorio agudo severo secundario a coronavirus 2 (SARS-CoV-2) fue definido como la relación $\mathrm{PaO}_{2} /$ $\mathrm{FiO}_{2}<200 \mathrm{mmHg}$ con PEEP $>5 \mathrm{cmH}_{2} \mathrm{O}$ en un paciente con RT-PCR para COVID-19 positiva. El posicionamiento en decúbito prono fue considerado en todos los pacientes con SARS-CoV-2 que persistieron con una relación $\mathrm{PaO}_{2} / \mathrm{FiO}_{2}<150 \mathrm{mmHg}$ posterior al bloqueo neuromuscular y a la titulación de la presión al final de la espiración con base en las mejores distensibilidades. El tiempo de inicio de la terapia prono fue considerado como el momento en que se realizó la terapia después de iniciada la ventilación mecánica, registrándose como «respondedor» a aquél que presentó un aumento sostenido (al menos por 24 horas) en la relación $\mathrm{PaO}_{2} / \mathrm{FiO}_{2}$ (> $150 \mathrm{mmHg}$ ) posterior al inicio de la terapia prono.

Límite de tiempo: Se realizó en el periodo comprendido del 21 de abril al 31 de julio de 2020.

Límite de espacio: Expedientes clínicos de pacientes con derechohabiencia que ingresaron a la Unidad de Cuidados Intensivos COVID de un hospital de tercer nivel de atención en México.

Análisis estadístico: Los pacientes fueron categorizados según su gravedad al momento del ingreso a terapia intensiva utilizando la escala SOFA, analizándose los factores de riesgo prevalentes mediante estadística descriptiva para después dar seguimiento a las variables fisiológicas y bioquímicas de cada uno de los pacientes durante el tiempo que permanecieron en cuidados intensivos. Una vez completada la base de datos, se calculó la probabilidad de supervivencia individual acumulada a lo largo del tiempo con el método de Kaplan Meier en pacientes con SARS-CoV-2 con base en el tratamiento con soporte multiorgánico aislado o soporte multiorgánico más terapia prono, mediante la prueba log rank se identificó si existía diferencia significativa de la supervivencia entre grupos. Se utilizó el programa SPSS versión 23.

Aspectos éticos: El estudio se registró en la Unidad de Educación e Investigación Médica con el número 055/20 y fue aprobado por los integrantes del Comité de Investigación en Salud y Ética en Investigación del hospital en la sesión ordinaria número 216, cuyo registro quedó asentado en el oficio 
207C0401010201S/JIC/214/2020. La aplicación del instrumento se realizó en las instalaciones del propio hospital, específicamente en el Servicio de Terapia Intensiva de acuerdo con los principios éticos establecidos en la decimoctava Asamblea Médica Mundial (Helsinki, 1964), la información fue de carácter confidencial, sin utilizar los nombres propios que contenían los expedientes elegidos. Por tratarse de un estudio de tipo observacional, sin ningún tipo de intervención, no requirió de consentimiento informado.

\section{RESULTADOS}

Durante el tiempo de estudio se reclutaron un total de 82 pacientes con diagnóstico de SARS-CoV-2, 59 hombres (72\%) y 23 mujeres (28\%), relación $2.5: 1$, la edad osciló entre los 25 y 65 años, con una media de 45.8 años y una mediana de 46.5 años; $47.6 \%$ de la población estudiada padecía algún grado de obesidad, $37.8 \%$ diabetes mellitus tipo 2, 15.9\% hipertensión arterial sistémica, $8.5 \%$ tenían antecedente de tabaquismo y $3.7 \%$ una enfermedad pulmonar previa. Es importante destacar que $12.2 \%$ de la población atendida correspondió a personal de salud. Con respecto a la gravedad con la que ingresaron a cuidados intensivos, la media en la puntuación de la escala SOFA fue de 5.8, con una mediana de seis y una desviación estándar de 1.639, con un mínimo de tres y un máximo de 11.

Del total de pacientes, $62(75.6 \%)$ requirieron terapia prono por una relación $\mathrm{PaO}_{2} / \mathrm{FiO}_{2}<150 \mathrm{mmHg}$ a pesar de la adecuada titulación de la presión al final de la espiración y del inicio del bloqueo neuromuscular, mientras que 20 pacientes $(24.4 \%)$ no fueron sometidos a esta terapia por presentar una relación $\mathrm{PaO}_{2} / \mathrm{FiO}_{2}>150 \mathrm{mmHg}$. En el seguimiento a 60 días se presentaron en el primer grupo 28 defunciones (con una supervivencia de $54.8 \%$ ) y cuatro en el segundo grupo (con una supervivencia de $80 \%$ ) ( $F i$ gura 1). La supervivencia acumulada entre los individuos de ambos grupos fue de $61 \%$ en el seguimiento a 60 días.

Dentro del grupo que requirió terapia prono, el tiempo promedio en que ocurrió la defunción fue de 42.6 días, mientras que en el grupo que no requirió terapia prono fue de 51.2 días, siendo para éstos el valor de $p$ de la prueba de log rank de 0.069 , la cual al ser mayor de 0.05 indica que no hay evidencia estadísticamente significativa para rechazar la hipótesis nula de igualdad de supervivencia en ambos grupos (el comportamiento de supervivencia es el mismo en ambos grupos).

Se analizó la supervivencia entre los pacientes que requirieron terapia prono con base en el tiempo en que esta terapia fue empleada, tomando como hora cero el momento en que se inició la ventilación mecánica, siendo aquellos en los que se inició la terapia dentro de las primeras 24 horas (43 pacientes) quienes tuvieron el mayor porcentaje de supervivencia a 60 días (55.8\%), seguido del grupo en que se inició la terapia prono entre los días 2-5 (15 pacientes) y en el grupo en que se inició después del sexto día (cuatro pacientes), con una supervivencia a 60 días de 53.3 y $50 \%$, respectivamente.

La defunción entre los pacientes con terapia prono en las primeras 24 horas se presentó a los 43.5 días en promedio, mientras que para los que iniciaron la terapia entre los días 2-5 el promedio fue a los 40.4 días y para el grupo con terapia prono después del día seis el promedio fue a los 41 días. En el análisis de supervivencia a 60 días, el valor de $p$ de la prueba de log rank fue de 0.326 , la cual al ser mayor de 0.05 indica que no hay evidencia estadísticamente significativa para rechazar la hipótesis nula de igualdad de supervivencia entre grupos (el comportamiento de supervivencia es el mismo entre grupos) (Figura 2).

A continuación, se analizó la supervivencia con base en la respuesta que se obtuvo tras haberse iniciado la terapia prono y fue contrastada con el grupo que no tuvo indicación de esta terapia. Como se describió previamente 20 pacientes tuvieron una relación $\mathrm{PaO}_{2} / \mathrm{FiO}_{2}$ $>150 \mathrm{mmHg}$, por lo que no fueron sometidos a terapia prono (la supervivencia en este grupo fue de $80 \%$ ); de los 62 pacientes con $\mathrm{PaO}_{2} / \mathrm{FiO}_{2}<150 \mathrm{mmHg}$ que recibieron terapia prono, 45 fueron catalogados como respondedores, grupo en el que se presentaron 14 defunciones (con una supervivencia calculada de $68.9 \%$ ); 17 pacientes fueron catalogados como no respondedores, grupo en el que ocurrieron 14 defunciones (con una supervivencia calculada de 17.6\%) (Figura 3).

Dentro del grupo que requirió terapia prono el tiempo promedio en que ocurrió la defunción fue de 42.6 días, mientras que en el subgrupo de quienes respondieron a la terapia el tiempo promedio fue de 48.5 días y en el subgrupo de quienes no respondieron a la terapia el

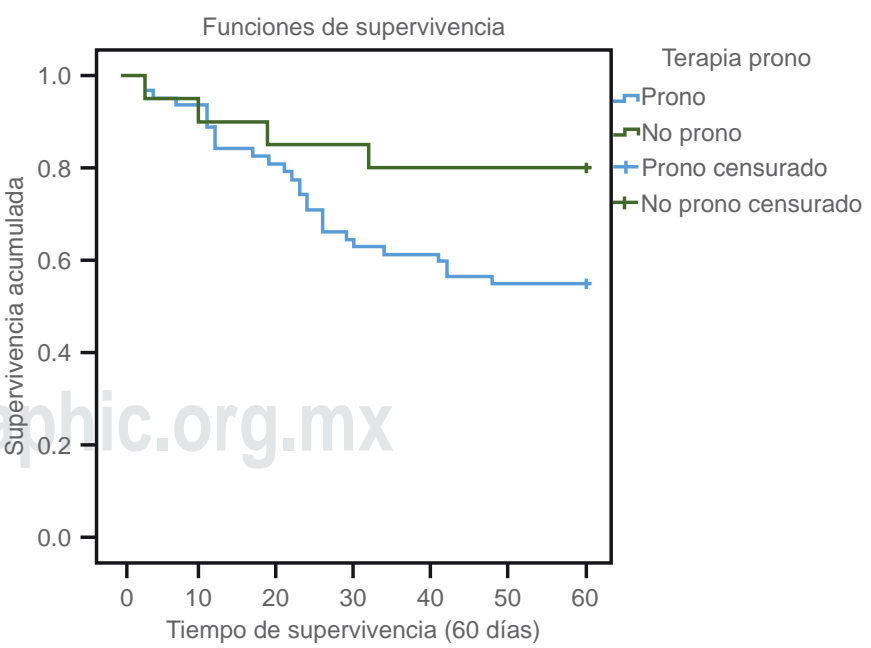

Figura 1: Curva de Kaplan Meier para la diferencia en la supervivencia entre grupos de pacientes con SARS-CoV-2 tratados con y sin terapia prono. Curva de Kaplan Meier que muestra menor supervivencia en el grupo de pacientes con indicación de terapia prono (azul) comparado con aquellos sin indicación de terapia prono (verde) $(p=0.069)$. 


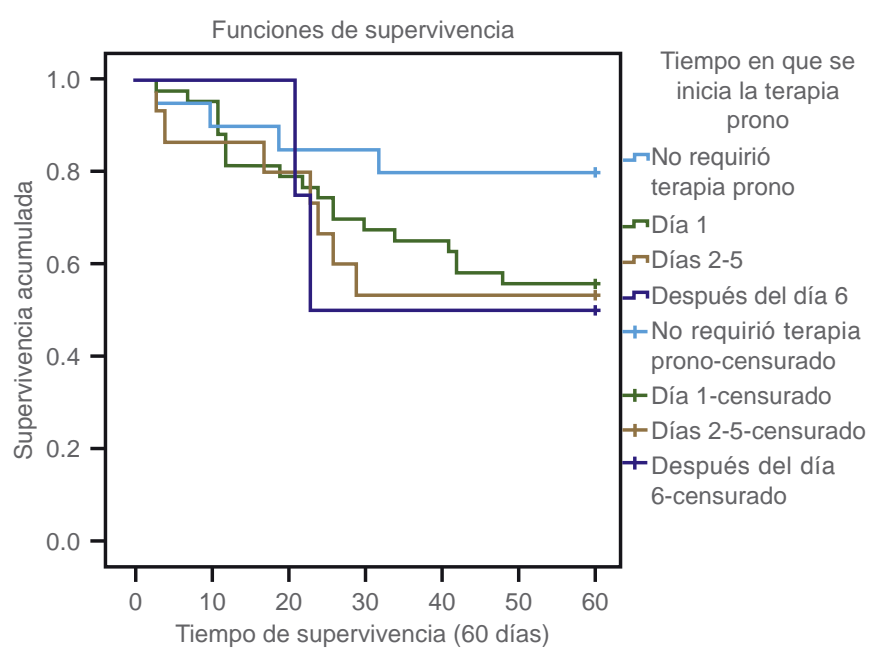

Figura 2: Curva de Kaplan Meier para la diferencia en la supervivencia entre grupos de pacientes con SARS-CoV-2 con base en el tiempo en que se inicia la terapia prono. Curva de Kaplan Meier que muestra menor supervivencia en el grupo de pacientes en quienes se inició la terapia prono después del día seis (morado) comparado con aquellos que no requirieron la terapia o fueron pronados dentro de los primeros cinco días (azul, verde, café) $(p=0.326)$.

tiempo promedio fue a los 27 días, siendo el valor de $p$ de la prueba de log rank de 0.000 , la cual al ser menor de 0.05 indica que sí hay evidencia estadísticamente significativa para rechazar la hipótesis nula de igualdad de supervivencia en ambos grupos (el comportamiento de supervivencia sí es distinto en ambos grupos).

Con respecto al análisis de supervivencia de acuerdo al número de veces en que fueron pronados los pacientes durante su estancia en terapia intensiva, el grupo que requirió un solo evento de prono (45 pacientes) tuvo una supervivencia a 60 días de $62.2 \%$, mientras que el grupo con dos eventos de prono (12 pacientes) tuvo una supervivencia de $50 \%$ y, finalmente, en aquellos que requirieron tres eventos de prono (cinco pacientes) la supervivencia fue de $0 \%$.

La defunción entre los pacientes con terapia prono durante una ocasión se presentó a los 44.9 días en promedio, mientras que para los que recibieron terapia prono en dos ocasiones el promedio fue a los 43.5 días y para el grupo con terapia prono en tres ocasiones el promedio fue a los 19.8 días. En el análisis de supervivencia a 60 días, el valor de $p$ de la prueba de log rank fue de 0.001, la cual indica que sí hay evidencia estadísticamente significativa para rechazar la hipótesis nula de igualdad de supervivencia entre grupos (el comportamiento de supervivencia sí es distinto entre grupos) (Figura 4).

\section{DISCUSIÓN}

Yu X. y su equipo ${ }^{10}$ reportaron 333 casos confirmados de COVID-19, la razón entre hombres (172, 51.7\%) y mujeres $(161,48.3 \%)$ fue de 1.07:1 con una mediana de edad de 50 años, siendo la edad concordante con lo reportado en nuestro estudio (46.5 años); sin embargo, con una proporción mayor de hombres con respecto a mujeres afectadas con una razón de 2.5:1. Además, fue de suma relevancia la gran diferencia encontrada en el número de trabajadores de la salud afectados donde ellos reportan que este grupo sólo representó $1.2 \%$ de los casos, contra $12.2 \%$ encontrado en nuestra población tratada.

En China, en el grupo de estudio de posición prono temprana, el objetivo fue explorar si esta estrategia podía mejorar de manera efectiva la hipoxia severa, el rendimiento de las imágenes de tomografía y el pronóstico de supervivencia de los pacientes con COVID-19 con hipoxia severa. ${ }^{11}$ Se reclutó un total de 60 pacientes, $23(38.3 \%)$ fueron colocados en decúbito prono de forma temprana y 37 (61.6\%) se mantuvieron en posición no prona, reportando que fallecieron $43.5 \%$ de los pacientes en el grupo en decúbito prono, en comparación con $75.7 \%$ en el grupo de posición no prona, observando en este punto la mayor diferencia con respecto a nuestros hallazgos, ya que la mortalidad en el grupo con estrategia prono fue de $45.2 \%$ con una supervivencia de $54.8 \%$, y para el grupo sin estrategia prono la mortalidad fue únicamente de $20 \%$, lo que representa una supervivencia de $80 \%$, lo cual enaltece el resultado de la cuidadosa selección de pacientes candidatos a la estrategia prono en combinación con el soporte multiorgánico, considerando que en nuestra población $75.6 \%$ fue sometida a estrategia prono y únicamente $24.4 \%$ se mantuvo en supino tras haber logrado una relación

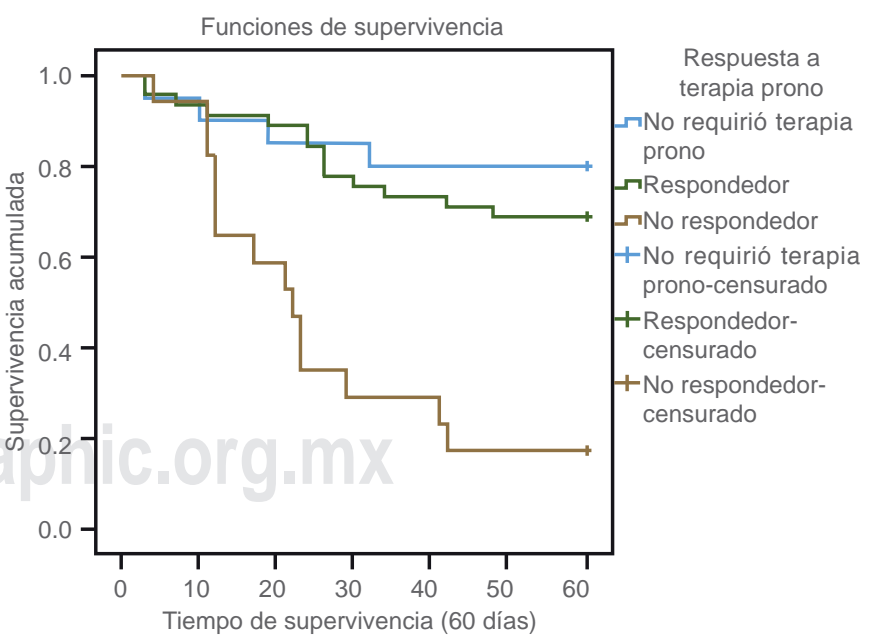

Figura 3: Curva de Kaplan Meier para la diferencia en la supervivencia entre grupos de pacientes con SARS-CoV-2 con base en la respuesta a la terapia prono. Curva de Kaplan Meier que muestra menor supervivencia en el grupo de pacientes no respondedores a prono (café), comparada con aquellos que no requirieron terapia prono o fueron respondedores a la terapia (azul y verde, respectivamente) $(p=0.000)$. 
$\mathrm{PaO}_{2} / \mathrm{FiO}_{2}>150 \mathrm{mmHg}$ con una adecuada titulación de la presión al final de la espiración y del inicio del bloqueo neuromuscular.

Por último, la mortalidad global tiene amplias variaciones en el mundo, en Wuhan osciló entre $86-97 \%$ entre los que requirieron ventilación mecánica, Reino Unido reportó que $67 \%$ de quienes requirieron ventilación mecánica murieron, los primeros reportes en Estados Unidos indicaban que $71-75 \%$ de los pacientes admitidos a la UCI que requirieron ventilación mecánica fallecieron, la mortalidad global entre pacientes con SARSCoV-2 que requirieron ventilación mecánica en nuestra unidad fue de $39 \%$ (supervivencia de $61 \%$ ), equiparable a la reportada por algunas unidades de cuidados intensivos también de Estados Unidos como en el estado de Atlanta, Georgia, donde la mortalidad fue de $35.7 \%$ en pacientes que requirieron ventilación mecánica e ingresaron a la $\mathrm{UCI}$ con una puntuación media en la escala de SOFA de siete puntos, similar a la media de nuestros pacientes a su ingreso (5.8). ${ }^{12}$

\section{CONCLUSIONES}

La terapia prono en pacientes con SARS-CoV-2 con una relación $\mathrm{PaO}_{2} / \mathrm{FiO}_{2}<150 \mathrm{mmHg}$ es una estrategia que permite mantener una supervivencia equiparable a aquella que tienen los pacientes que ingresan con una $\mathrm{PaO}_{2} / \mathrm{FiO}_{2}$ superior a $150 \mathrm{mmHg}(\mathrm{p}=0.069)$.

La mayor supervivencia ocurre entre los pacientes en quienes se realiza la terapia prono dentro de las primeras 24 horas; sin embargo, no hay una diferencia signi-

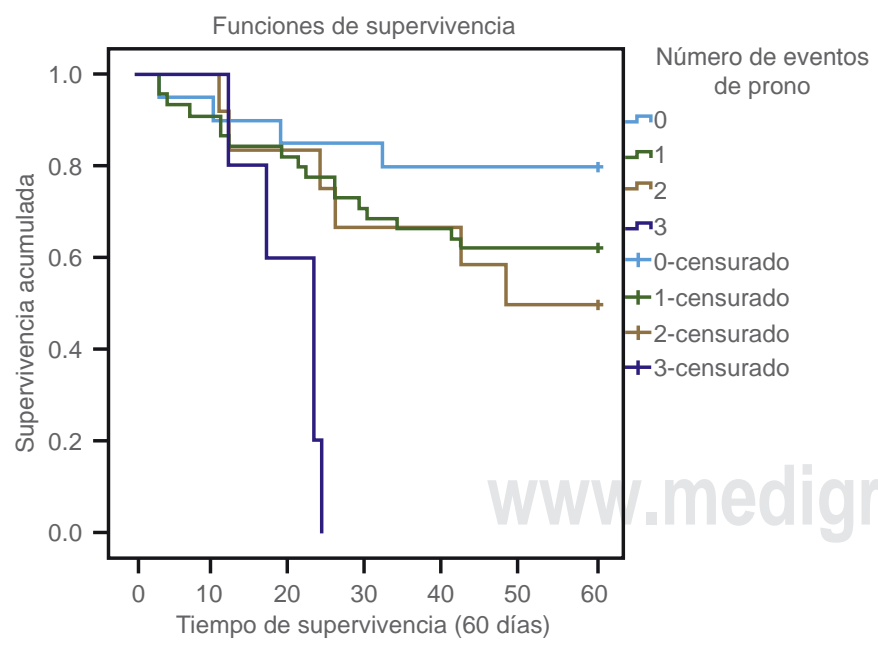

Figura 4: Curva de Kaplan Meier para la diferencia en la supervivencia entre grupos de pacientes con SARS-CoV-2 con base en el número de terapias prono que recibieron. Curva de Kaplan Meier que muestra menor supervivencia en el grupo de pacientes que recibieron terapia prono en tres ocasiones (morado), comparada con aquellos que no requirieron terapia prono o fueron sometidos a la terapia en una o dos ocasiones (azul, verde y café, respectivamente) $(p=0.001)$. ficativa con respecto a la terapia que se inicia después de este tiempo $(p=0.326)$. Por otra parte, la supervivencia es significativamente mayor en aquellos que responden a esta terapia en comparación con quienes no $(p=0.000)$, mientras que requerir más de un evento de prono se asocia a menor supervivencia $(p=0.001)$.

\section{REFERENCIAS}

1. WHO. Clinical management of severe acute respiratory infection when Novel coronavirus ( $\mathrm{nCoV}$ ) infection is suspected: interim guidance. Jan 11, 2020.

2. Chen N, Zhou M, Dong X, Qu J, Gong F, Han Y, et al. Epidemiological and clinical characteristics of 99 cases of 2019 novel coronavirus pneumonia in Wuhan, China: a descriptive study. Lancet. 2020;395(10223):507-513.

3. Ruan Q, Yang K, Wang W, Jiang L, Song J. Clinical predictors of mortality due to COVID-19 based on an analysis of data of 150 patients from Wuhan, China. Intensive Care Med. 2020;46(5):846-848.

4. Matthay MA, Zemans RL, Zimmerman GA, Arabi YM, Beitler JR, Mercat A, et al. Acute respiratory distress syndrome. Nat Rev Dis Primers. 2019;5(1):18.

5. Guérin C, Reignier J, Richard JC, Beuret P, Gacouin A, Boulain $\mathrm{T}$, et al. Prone positioning in severe acute respiratory distress syndrome. N Engl J Med. 2013;368(23):2159-2168.

6. Beitler JR, Shaefi S, Montesi SB, Devlin A, Loring SH, Talmor D, et al. Prone positioning reduces mortality from acute respiratory distress syndrome in the low tidal volume era: a meta-analysis. Intensive Care Med. 2014;40(3):332-341.

7. Gattinoni L, Taccone P, Carlesso E, Marini JJ. Prone position in acute respiratory distress syndrome. Rationale, indications, and limits. Am J Respir Crit Care Med. 2013;188(11):1286-1293.

8. Fan E, Del Sorbo L, Goligher EC, Hodgson CL, Munshi L, Walkey AJ, et al. An Official American Thoracic Society/ European Society of Intensive Care Medicine/Society of Critical Care Medicine Clinical Practice Guideline: mechanical ventilation in adult patients with acute respiratory distress syndrome. Am J Respir Crit Care Med. 2017;195(9):1253-1263.

9. Guérin C, Beuret P, Constantin JM, Bellani G, Garcia-Olivares $\mathrm{P}$, Roca $\mathrm{O}$, et al. A prospective international observational prevalence study on prone positioning of ARDS patients: the APRONET (ARDS Prone Position Network) study. Intensive Care Med. 2018;44(1):22-37.

10. Yu X, Sun X, Cui P, Pan H, Lin S, Han R, et al. Epidemiological and clinical characteristics of 333 confirmed cases with coronavirus disease 2019 in Shanghai, China. Transbound Emerg Dis. 2020;67(4):1697-1707.

11. Zang X, Wang Q, Zhou H, Liu S, Xue X; COVID-19 Early Prone Position Study Group. Efficacy of early prone position for COVID-19 patients with severe hypoxia: a single-center prospective cohort study. Intensive Care Med. 2020;46(10):19271929.

12. Auld SC, Caridi-Scheible M, Blum JM, Robichaux C, Kraft C, Jacob JT, et al. ICU and ventilator mortality among critically ill adults with coronavirus disease 2019. Crit Care Med. 2020;48(9):e799-e804.

Patrocinios: Ninguno.

Relación de conflicto de intereses: Los autores declaran no tener conflicto de intereses.

Correspondencia:

Netzahualcóyotl González Pérez

Av. Baja Velocidad Núm. 284,

San Jerónimo Chicahualco, 52170,

Toluca de Lerdo, México.

Teléfono: 72227563 00, ext. 1061534 y 1061535

E-mail: netza2014@outlook.com 